\title{
Efficient Sensitivity Calculations for Optimization of Power Delivery Network Impedance
}

\author{
A. Ege Engin, Member, IEEE
}

\begin{abstract}
This paper presents efficient algorithms for sensitivity calculations of power delivery network (PDN) impedance. The sensitivity algorithm is based on the adjoint system method. Efficient calculation of sensitivity is critical for optimization of complex PDNs. The frequency-domain design of a PDN is a multiobjective optimization problem, which is formulated as a minimax problem in this paper. The design variables are typically the decoupling capacitors (decaps). The objective function is the input impedance of the PDN calculated at relevant frequencies. This paper also presents an efficient algorithm for large-change sensitivity calculations. Based on this method, what-if scenarios can be simulated efficiently, such as the addition, modification, or relocation of a few decaps or a small change in the geometry.
\end{abstract}

Index Terms-Decoupling, ground bounce, power delivery network (PDN), power integrity optimization, simultaneous switching noise, target impedance, Woodbury formula.

\section{INTRODUCTION}

$\mathbf{T}$ HE CLOCK speed of a computer system is beginning to be limited by the power supply noise, which needs to be controlled not only at the transistor level, but also at the system level. Even though transistors can operate at a high frequency, reliable operation of a computer system is possible only at a lower frequency because of power supply noise. This presents a system-level design problem as the power delivery network (PDN) connects the whole system together from the integrated circuit (IC) to the voltage regulator on the printed circuit board (PCB). In addition to this power integrity problem, power supply noise may also cause severe electromagnetic interference problems because of edge radiation from the boundaries of the power/ground planes [1]. Predicting and reducing the power supply voltage noise, or in other words, improving the power integrity of the design is, therefore, crucial. As the technology progresses, power integrity needs to be maintained in spite of lower noise requirements with decreasing supply voltages below $1 \mathrm{~V}$, and increasing complexity of PDNs with introduction of new IC packaging options such as 3-D integration and embedded components. Hence, fast and accurate PDN optimization algorithms are needed.

The maximum power-supply voltage ripple specification is commonly converted to another specification in the frequency

Manuscript received August 21, 2009; revised January 4, 2010. First published March 4, 2010; current version published May 19, 2010.

The author is with the Department of Electrical and Computer Engineering, San Diego State University, San Diego, CA 92182 USA (e-mail: aengin@mail.sdsu.edu).

Color versions of one or more of the figures in this paper are available online at http://ieeexplore.ieee.org.

Digital Object Identifier 10.1109/TEMC.2010.2042059 domain: target impedance, which sets the upper bound on the magnitude of the PDN impedance as seen by the IC [2]. A PDN design that meets the target impedance in frequency domain does not necessarily guarantee that the time-domain voltage ripple is below a predetermined threshold value [3]. On the other hand, minimizing the time-domain power-supply voltage ripple as an optimization goal would require the knowledge of the full details of the IC and the current signature [4]. As the time-domain current signature is difficult to extract through measurements or simulations, the target-impedance specification is commonly preferred in system-level PDN design and will be applied in this paper.

To optimize the PDN, the most significant variables are the selection and location of decoupling capacitors (decaps) [5]. By iteratively changing these parameters, the designer targets at reducing the impedance by controlling the resonances of the PDN. This is a big design challenge because of the resonances caused by the interaction between decaps. The power/ground planes also generate a complicated impedance profile with multiple resonances. Current methodologies for system-level PDN design are based on stochastic optimization algorithms such as genetic algorithms [6] and simulated annealing [7], [8]. With the proposed sensitivity algorithms in this paper, a gradientbased optimization approach becomes possible for system-level PDN optimization. We demonstrate the calculation of sensitivities using the adjoint system method and apply the minimax optimization method to reduce the magnitude of the input impedance below a frequency-dependent target impedance profile. Gradient-based optimization methods have been previously applied for the time-domain optimization of the power grid on an IC [9]. Also, this paper presents a fast large-change sensitivity approach, or an incremental simulation approach to rapidly simulate multiple PDN designs with changes in a few parameters.

This paper is organized as follows. In Section II, the finitedifference method for analysis of power/ground planes is briefly explained. The new sensitivity algorithm is then introduced based on this method. Section III introduces the minimax approach for optimization of the PDNs. The gradient information for the minimax algorithm is computed efficiently using the sensitivity algorithms in Section II. Section IV introduces a new method for fast resimulation of a design after a change has been made either in the geometry of the power/ground planes or in the locations or values of decaps. This method for fast design space exploration is based on the large-change sensitivity analysis in circuit design. Large-change sensitivity analysis allows arbitrary variation of chosen variables, whereas the sensitivity algorithm in Section II merely provides the gradient information 


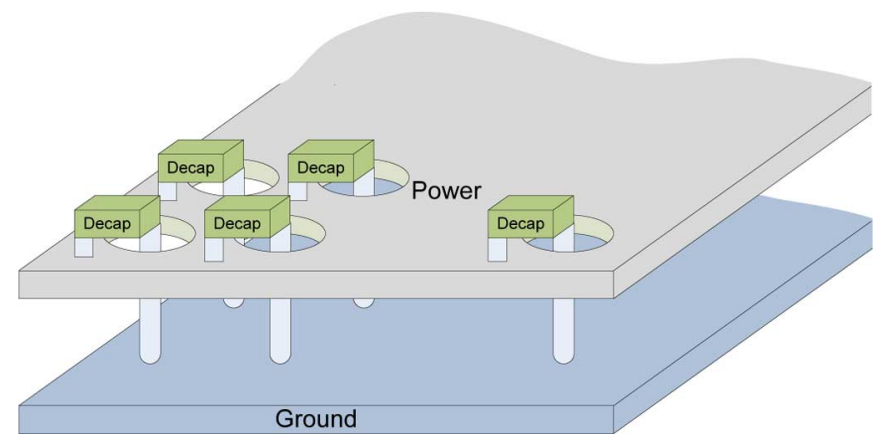

Fig. 1. Power/ground planes separated by a thin dielectric and populated with decaps connected with vias.

for a small change in the given variables. This approach can, in principle, also be used within an optimization algorithm as in Section III, where multiple similar designs need to be simulated at each iteration of the optimization algorithm.

\section{Sensitivity CALCUlations FOR PDNs}

\section{A. Analysis of Power/Ground Planes and}

\section{Decoupling Capacitors}

Consider the power/ground planes on a chip package or PCB, as shown in Fig. 1. An efficient way of calculating the impedance profile of this structure can be developed based on the 2-D finite-difference method [10], [11]. We make use of the fact that the thickness of the dielectric is much smaller than the lateral dimensions, hence, the variation of the electric and magnetic fields with respect to the vertical $z$-direction can be neglected. Then, assuming there are no decaps for the time being, the voltage $u(x, y)$ at any point $P(x, y)$ can be described with the 2-D Helmholtz wave equation

$$
\nabla^{2} u+k^{2} u=-j J_{z} \omega \mu d
$$

where $k$ is the wavenumber, $J_{z}$ is the current density in the vertical direction, $\omega$ is the angular frequency, $\mu$ is the permeability, and $d$ is the dielectric thickness. The boundary conditions are of Neumann type for the planes, which can have arbitrary borders or cutouts.

In this section, the finite-difference method for the numerical solution of (1) is briefly explained as it will be useful to present the sensitivity algorithms in the following sections. The details of the finite-difference method can be found, for example, in [10]-[12]. The five-point finite-difference discretization of the Laplace operator $\left(\nabla^{2}\right)$ in (1) yields

$\frac{u_{i, j+1}+u_{i+1, j}+u_{i, j-1}+u_{i-1, j}-4 u_{i, j}}{-j \omega \mu d}+j \omega \varepsilon \frac{h^{2}}{d} u_{i, j}=I_{z}$

at each discretization point $(i, j)$, where $I_{z}$ represents any current sources connected to a unit cell with a mesh length of $h$, and $\varepsilon$ is the permittivity. It is useful to interpret this approximation using an equivalent circuit representation, as shown in Fig. 2,

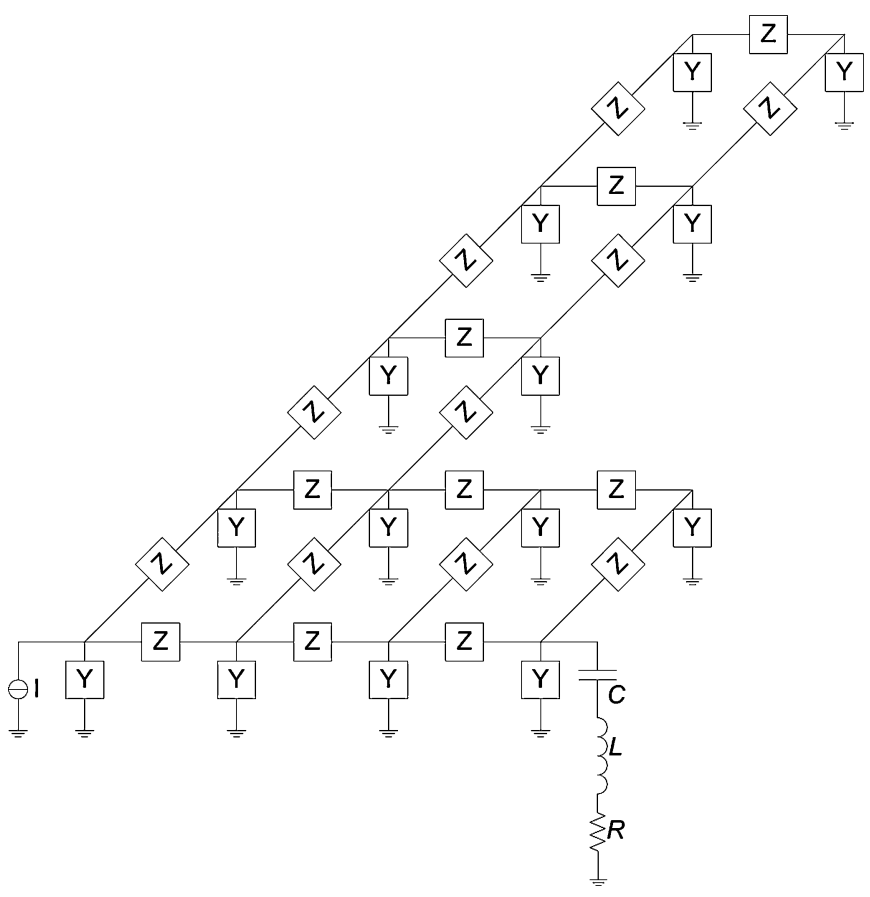

Fig. 2. Equivalent circuit representation for the example of an L-shaped power/ground plane pair with a current source connected to the lower left node and a decap connected to the lower right node.

where

$$
\begin{aligned}
& Z=j \omega \mu d \\
& Y=j \omega \varepsilon \frac{h^{2}}{d}
\end{aligned}
$$

for the lossless case. For practical power/ground planes, the conductor and substrate losses can be considered as

$$
\begin{aligned}
& Z=j \omega \mu d+2 \sqrt{\frac{j \omega \mu}{\sigma}} \operatorname{coth}(t \sqrt{j \omega \mu \sigma}) \\
& Y=j \omega \varepsilon(1-j \tan \delta) \frac{h^{2}}{d}
\end{aligned}
$$

where $\sigma$ is the conductivity of the planes, $t$ is the thickness of each plane, and $\tan \delta$ is the loss tangent of the dielectric [12]. The nodal admittance matrix $\overline{\bar{Y}}$ of the equivalent circuit model in Fig. 2 can be built up as

$$
\overline{\bar{Y}} \bar{V}=\bar{I}
$$

where $\bar{V}$ is the vector of node voltages and $\bar{I}$ is the vector of any connected current sources. Solution of this matrix equation provides the distribution of voltage for any current excitation, which is equivalent to the self and transfer impedances assuming a current excitation of $1 \mathrm{~A}$ at a single node. To build up $\overline{\bar{Y}}$, each shunt admittance at node $p$ is added as

$$
\left[\begin{array}{ccc}
\ddots & & \\
\cdots & Y & \cdots \\
& & \ddots
\end{array}\right]\left[\begin{array}{c}
\vdots \\
V_{p} \\
\vdots
\end{array}\right]=\left[\begin{array}{c}
\vdots \\
I_{p} \\
\vdots
\end{array}\right]
$$


whereas each series impedance between nodes $p$ and $q$ is added as

$$
\left[\begin{array}{ccccc}
\ddots & & & & \\
\cdots & \frac{1}{Z} & \cdots & -\frac{1}{Z} & \cdots \\
& & \ddots & & \\
\cdots & -\frac{1}{Z} & \cdots & \frac{1}{Z} & \cdots \\
& & & & \ddots
\end{array}\right]\left[\begin{array}{c}
\vdots \\
V_{p} \\
\vdots \\
V_{q} \\
\vdots
\end{array}\right]=\left[\begin{array}{c}
\vdots \\
I_{p} \\
\vdots \\
I_{q} \\
\vdots
\end{array}\right] .
$$

The decaps are simply included in the matrix as additional shunt admittances with the admittance $1 /[1 /(j \omega C)+j \omega L+$ $R]$, based on a series $R L C$ model for the decap. Hence, the addition of a decap merely corresponds to the addition of its admittance on the corresponding diagonal element of the nodal admittance matrix as in (8).

\section{B. Sensitivity Calculation}

Next, we show an efficient method to calculate the sensitivity of the input impedance with respect to a decap connecting a power plane with a ground plane. To calculate the sensitivity of the input impedance, we can employ the adjoint system method [13]. To this end, taking the derivative of (7) with respect to the decap $C_{j}$ yields

$$
\frac{\partial \overline{\bar{Y}}}{\partial C_{j}} \bar{V}+\overline{\bar{Y}} \frac{\partial \bar{V}}{\partial C_{j}}=\frac{\partial \bar{I}}{\partial C_{j}}
$$

or

$$
\frac{\partial \bar{V}}{\partial C_{j}}=-\overline{\bar{Y}}^{-1}\left(\frac{\partial \overline{\bar{Y}}}{\partial C_{j}} \bar{V}-\frac{\partial \bar{I}}{\partial C_{j}}\right) .
$$

In order to calculate the input impedance at node $i$, the current vector $\bar{I}$ is set to a unit column vector, whose elements are zero except the $i$ th element that is equal to 1 . The solution of the matrix equation in (7) then yields the input impedance at the $i$ th element $V_{i}$ of the solution vector, which can be obtained as

$$
V_{i}=\bar{d}^{T} \bar{V}
$$

where the superscript $T$ denotes the transpose and $\bar{d}$ is a constant unit vector that is exactly same as the current vector $\bar{I}$ used to calculate the impedance. The sensitivity of the input impedance at node $i$ with respect to the decap $C_{j}$ can then be calculated as

$$
\frac{\partial V_{i}}{\partial C_{j}}=\bar{d}^{T} \frac{\partial \bar{V}}{\partial C_{j}}=-\bar{d}^{T} \overline{\bar{Y}}^{-1}\left(\frac{\partial \overline{\bar{Y}}}{\partial C_{j}} \bar{V}-\frac{\partial \bar{I}}{\partial C_{j}}\right) .
$$

The adjoint vector $\overline{V^{a}}$ is defined as

$$
{\overline{V^{a}}}^{T}=-\bar{d}^{T} \overline{\bar{Y}}^{-1}
$$

or equivalently

$$
\overline{\bar{Y}}^{T} \overline{V^{a}}=-\bar{d} .
$$

The adjoint vector needs to be calculated only once independent of the number of decaps. Moreover, the matrix equation in (15) can be solved efficiently as the LU decomposition of $\overline{\bar{Y}}$ is already available from the solution of (7). Note that $\overline{\bar{Y}}$ is a symmetric matrix for the 2-D finite-difference approximation [12]. As the goal function is the input impedance, the vectors $\bar{d}$ and $\bar{I}$ are equivalent as noted earlier. So, the adjoint vector is actually already available for this special case, since $\overline{V^{a}}=-\bar{V}$. In analysis of noise coupling, the transfer impedance might be of consideration. In that case, the vectors $\bar{d}$ and $\bar{I}$ would be different.

Considering that the current $\bar{I}$ is a constant vector, the sensitivity of the input impedance $Z_{i}=V_{i}$ with respect to the decap $C_{j}$ in (13) can be simplified as

$$
\frac{\partial Z_{i}}{\partial C_{j}}=\frac{\partial V_{i}}{\partial C_{j}}=-\bar{V}^{T} \frac{\partial \overline{\bar{Y}}}{\partial C_{j}} \bar{V} .
$$

The partial derivative $\partial \overline{\bar{Y}} / \partial C_{j}$ has only one nonzero entry (or four nonzero entries in the rare case of a series capacitor), therefore, the calculation of the sensitivity using (16) comes at negligible cost. In PDN design, the goal function is the magnitude of the input impedance, whose sensitivity with respect to the decap $C_{j}$ can be obtained from (16) as

$$
\frac{\partial\left|Z_{i}\right|}{\partial C_{j}}=\Re\left\{\frac{\left|Z_{i}\right|}{Z_{i}} \frac{\partial Z_{i}}{\partial C_{j}}\right\}=\Re\left\{-\frac{\left|Z_{i}\right|}{Z_{i}} \bar{V}^{T} \frac{\partial \overline{\bar{Y}}}{\partial C_{j}} \bar{V}\right\}
$$

where $\Re$ takes the real part of its argument.

The derivative of $\overline{\bar{Y}}$ in (17) consists of all zero elements except at the $j$ th diagonal element. Assuming an $R L C$ model for the capacitor $C_{j}$, (17) can then be simplified as

$$
\frac{\partial\left|Z_{i}\right|}{\partial C_{j}}=\Re\left\{-\frac{j \omega}{[1+j \omega C(j \omega L+R)]^{2}} \frac{\left|V_{i}\right|}{V_{i}} V_{j}^{2}\right\} .
$$

Once (7) is solved for the input impedance, all the elements in (18) are available. Hence, the sensitivity with respect to an existing decap is obtained at negligible cost. Calculation of the sensitivity with respect to $N$ decaps has only an $O(N)$ complexity.

\section{Numerical Example for Sensitivity}

This example demonstrates the impedance sensitivity of a pair of power/ground planes with respect to a decap. The sensitivity is calculated using (18). The planes have a square shape of size $20 \mathrm{~cm} \times 20 \mathrm{~cm}$. The two plates are separated by a dielectric with thickness of $200 \mu \mathrm{m}$, dielectric constant of 4 , and loss tangent of 0.02 . The plates are made of $35-\mu \mathrm{m}$-thick copper. The IC is assumed to be in the center of the board, so the input impedance is calculated at the center node. A decap with an $R L C$ model of $C=100 \mathrm{nF}, L=0.2 \mathrm{nH}$, and $R=10 \mathrm{~m} \Omega$ is placed in one corner of the board.

Fig. 3(a) shows the input impedance calculated for this example. The sensitivity is shown in Fig. 3(b). To verify the adjoint system method, the sensitivity has also been calculated using centered finite differences. The agreement is excellent. At frequencies below the first series resonance at about $15 \mathrm{MHz}$, the sensitivity is negative, suggesting that increasing the capacitance would help in decreasing the impedance. This is expected, as at such low frequencies the decoupling capacitance 


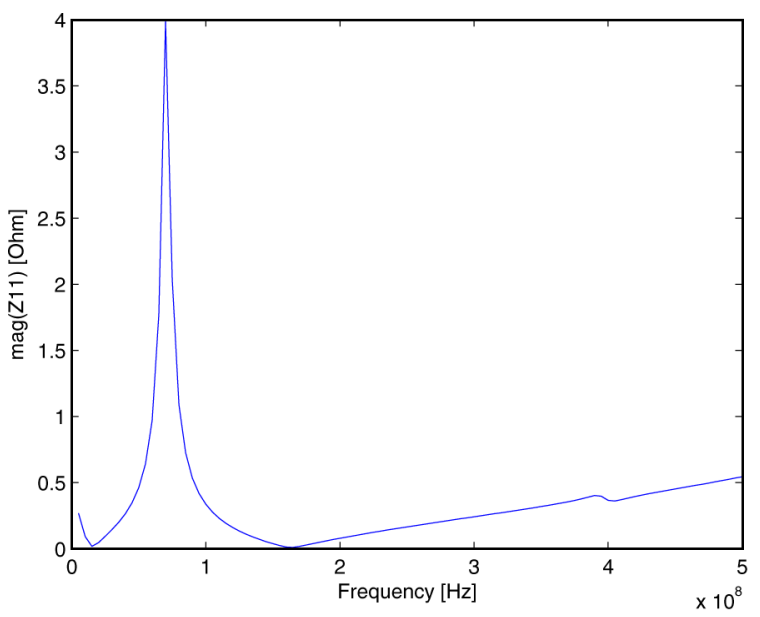

(a)

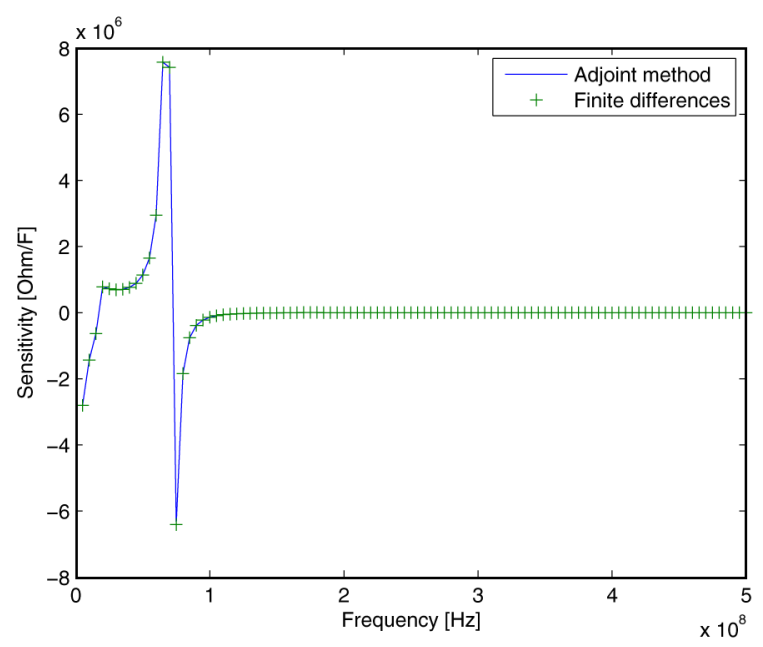

(b)

Fig. 3. (a) Input impedance at the center of a square-shaped ( $20 \mathrm{~cm}$ on a side) board with a decap in one corner. (b) Sensitivity calculated using the proposed adjoint system method and standard centered finite differences.

is dominant and inductances can be neglected. The sensitivity then changes the polarity around the resonance frequencies. The input impedance is not very sensitive to the decoupling capacitance above approximately $100 \mathrm{MHz}$. This, however, does not directly imply that the decap is ineffective above $100 \mathrm{MHz}$. Even though the impedance may be insensitive to a small change in the capacitance, the parasitic inductance and resistance of the capacitor may still be influencing the impedance. Nevertheless, the sensitivity provides useful information to decide which decaps to optimize in order to decrease the impedance at a given frequency range.

\section{OPTIMIZATION OF PDN IMPEDANCE}

PDN design is typically accomplished in the frequency domain, where the goal is to lower the impedance resulting in less power supply voltage ripple. Power/ground planes are used to establish a low-impedance path between the decaps on a chip package or PCB and ICs. The interaction of various

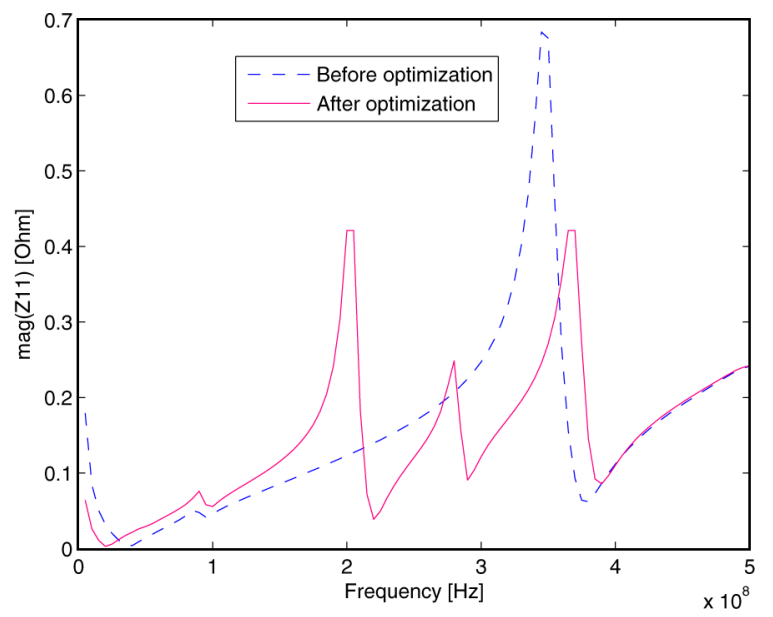

Fig. 4. Minimax optimization of the PDN impedance including 16 decaps.

decaps, however, cause undesirable antiresonances causing high impedance peaks. Decoupling capacitors also interact with the power/ground planes causing additional resonances. Hence, a major goal of the PDN design process is to find the optimum values for decaps, resulting in low-impedance profile.

The objective function is typically defined as the impedance seen by the IC, looking into the power and ground terminals. The magnitude of this impedance at all frequencies of interest is desired to be below the target impedance. Assume the impedance $Z_{j}$ is simulated at $m$ distinct frequencies $f_{j}, j=1,2, \ldots, m$. The target impedance is also provided at the same frequency

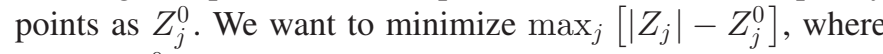
$Z_{j}$ and $Z_{j}^{0}$ are the impedance and target impedance at the $j$ th frequency, respectively. Hence, we formulate the optimization as a minimax problem. The design variables are the decaps, which are defined with the vector $\bar{h}$. Formally, we then define the problem as

$$
\text { Minimize }: F(\bar{h})=\max _{j}\left[\left|Z_{j}(\bar{h})\right|-Z_{j}^{0}\right]
$$

subject to the inequality constraints that the capacitance values should be in a valid range. General-purpose optimization algorithms can now be applied to find the optimum variables yielding the minimum impedance. The optimization algorithm we apply is based on the sequential quadratic programming method [14] in MATLAB. We calculate the sensitivity of the impedance with respect to the decaps as presented in Section II.

\section{A. Numerical Example for Optimization}

The same power/ground plane pair as in previous example on sensitivity is used for this example on optimization of the PDN impedance. The thickness of the dielectric is reduced to $100 \mu \mathrm{m}$, and 16 decaps are evenly distributed on the planes. The inductance of the capacitors is $0.2 \mathrm{nH}$, the resistance is $10 \mathrm{~m} \Omega$. The capacitance values are constrained to be between 1 and $200 \mathrm{nF}$. The starting values for all the decaps are set to $10 \mathrm{nF}$. For this problem, the minimax algorithm converges to a minimum after 81 iterations for a total of 100 frequency points between 5 and $500 \mathrm{MHz}$. Fig. 4 shows the input impedance 


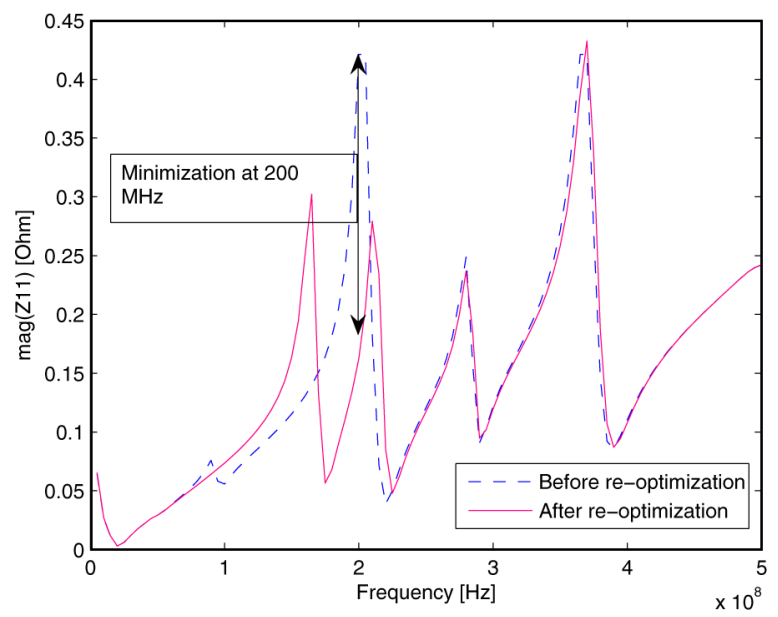

Fig. 5. Reoptimization to further reduce the impedance at $200 \mathrm{MHz}$.

at the center of the board for the initial setup and after values of the decaps have been optimized. In this example, a flat target impedance has been assumed. A reduction of $38 \%$ in the maximum magnitude of the input impedance has been achieved after optimization. The optimized values of the decaps ranged between 1 and $50 \mathrm{nF}$. Even though the variation of decap values has resulted in additional resonances in the impedance profile, the maximum impedance value has been reduced using the proposed optimization methodology.

Next, assume that there is a clock harmonic at $200 \mathrm{MHz}$ and it is desired to further reduce the impedance at that particular frequency. To approach this problem, we can restart the optimization algorithm with the optimized values obtained in the previous step. In order to emphasize the minimization objective at $200 \mathrm{MHz}$, we define the target impedance at this frequency to be very low. It is taken as zero in this example. We define the target impedance at other frequencies to be equal to the optimized solution in order not to excessively increase the impedance at other frequency points. After 11 iterations, the optimized solution in Fig. 5 is obtained. The impedance at $200 \mathrm{MHz}$ has been reduced to $0.16 \Omega$ from $0.42 \Omega$. Note that the improvement at $200 \mathrm{MHz}$ comes at an expense at some other frequencies. However, the minimax objective function has been reduced by $62 \%$.

\section{FAST DESIGN Space EXPLORATION}

The sensitivity-based approach described in Section II is useful in finding the trend or the gradient of the impedance as one slightly modifies decaps on the PCB. On the other hand, accurate simulation of what-if scenarios requires large changes in the parameters, which cannot be predicted using the differential sensitivity. The simulation of the entire setup starting from scratch is required, even though only a single decap may be removed, added, modified, or moved to another location on the PCB. The previous simulation results are not used to expedite this process. Next, we describe a novel technique to eliminate this problem by allowing an incremental simulation of the same PDN after a change in a few of its parameters.

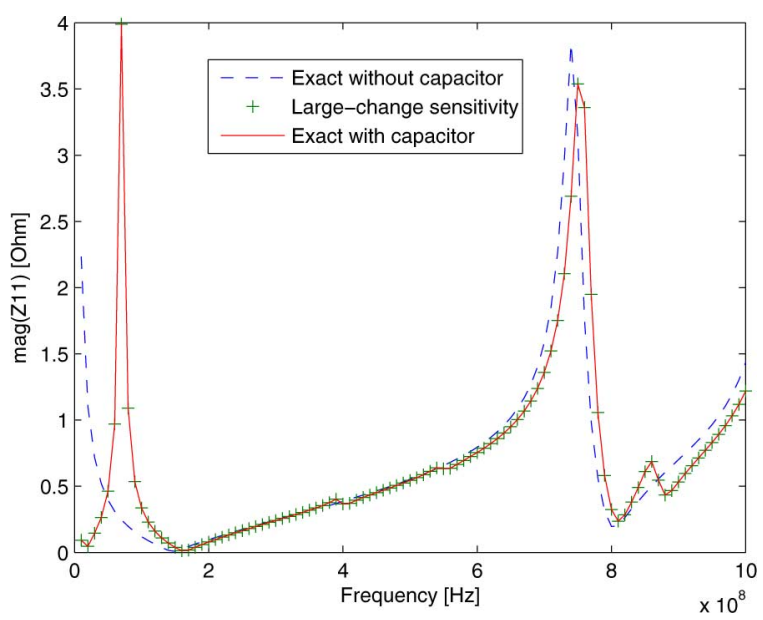

Fig. 6. Application of the Woodbury formula for rapid calculation of the impedance profile with the addition of a decap.

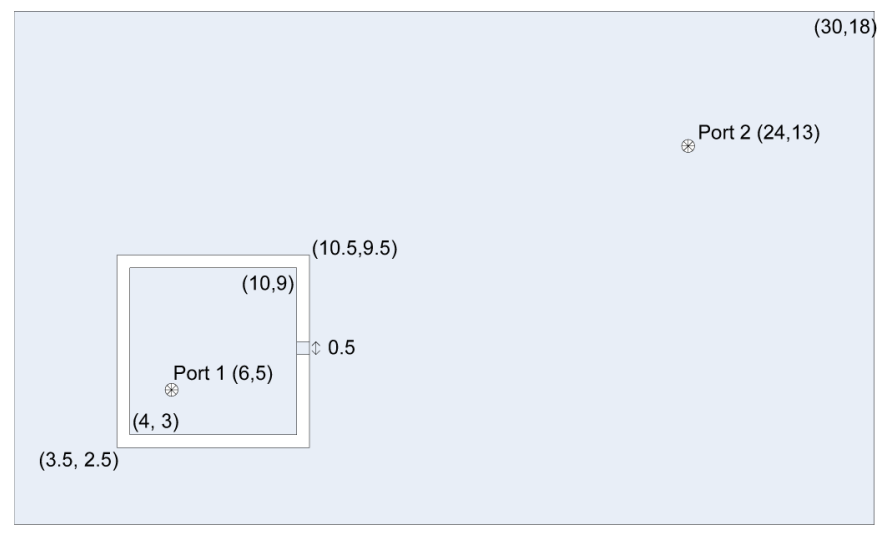

Fig. 7. Geometry of the power island and the bridge with a width of $0.5 \mathrm{~mm}$ connected to the center of the power island's right edge. All units are in millimeters.

The proposed method is based on the large-change sensitivity approach used in circuit theory, or mathematically, the application of the Woodbury formula [15]. This novel approach allows fast design space exploration of PDNs by efficient simulation of what-if scenarios.

Assume that the matrix equation in (7) has been solved for the nominal case. Now, it is desired to obtain the solution of a new simulation setup with a change in a few of the PDN parameters. So, the new equation to be solved has the form

$$
(\overline{\bar{Y}}+\overline{\bar{K}}) \bar{V}=\bar{I}
$$

where the equation

$$
\overline{\bar{Y} V_{0}}=\bar{I}
$$

has already been solved by the LU decomposition. The Woodbury formula works if the matrix $\overline{\bar{K}}$ can be expressed as a product of two matrices yielding $\overline{\bar{K}}=\overline{\bar{U}} \overline{\bar{M}}^{T}$. The updated solution $\bar{V}$ can be obtained by solving the additional system

$$
\overline{\bar{Y}} \overline{\bar{Z}}=\overline{\bar{U}}
$$




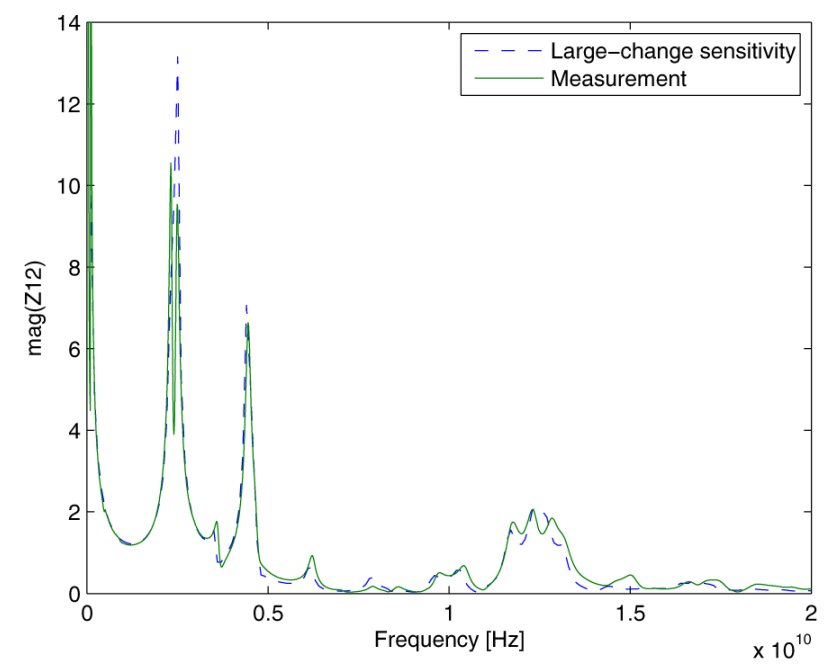

(a)

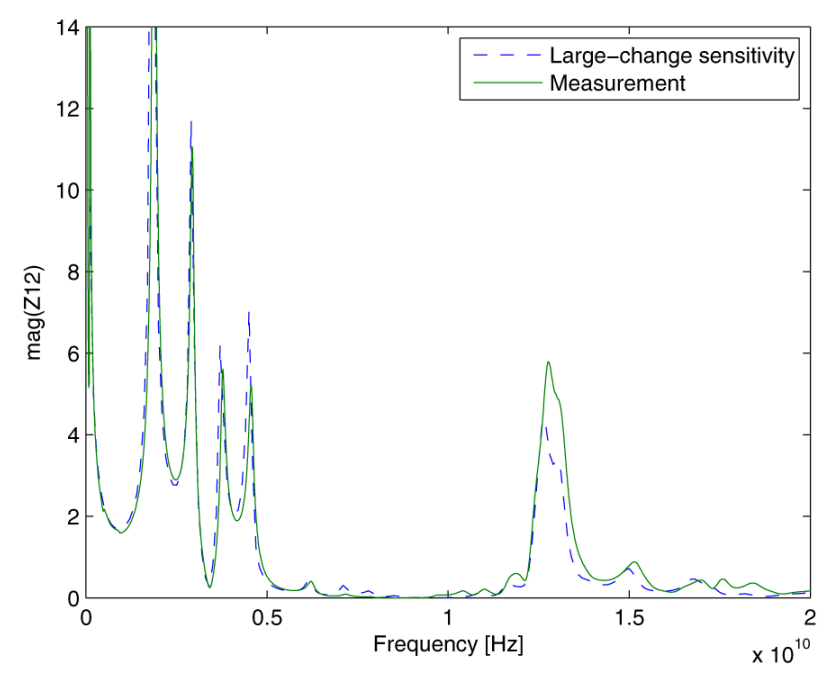

(c)

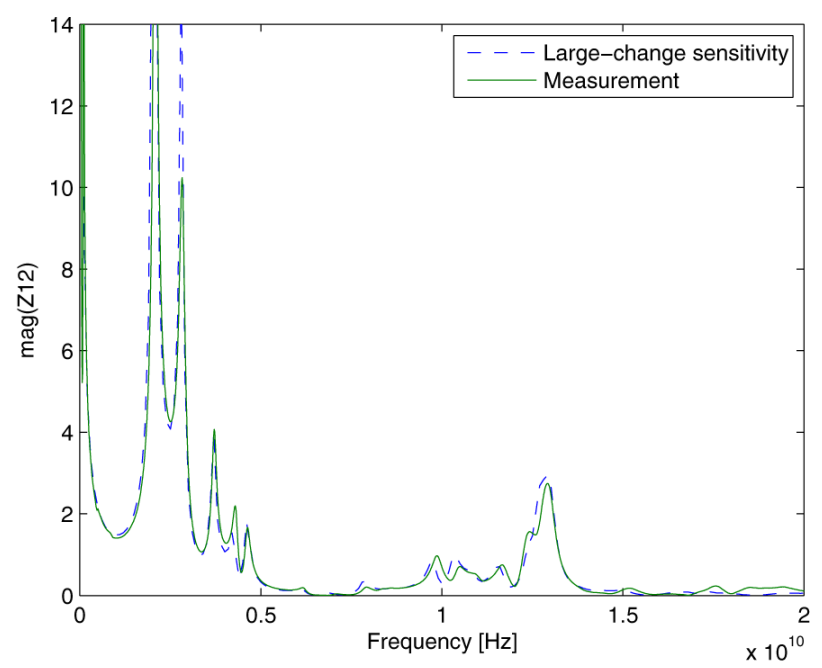

(b)

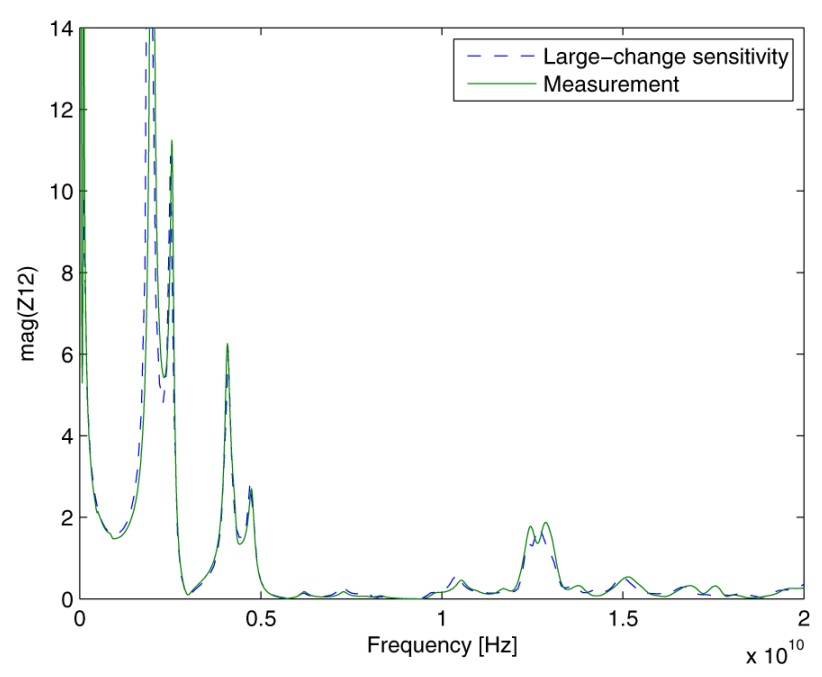

(d)

Fig. 8. Measured and simulated transfer impedance using the large-change sensitivity algorithm, when the bridge is connected to the power island at the center of its (a) right, (b) top, (c) left, and (d) bottom edge.

and calculating the matrix $\overline{\bar{H}}$

$$
\overline{\bar{H}}=\left(\overline{\overline{1}}+\overline{\bar{M}}^{T} \overline{\bar{Z}}\right)^{-1}
$$

yielding the desired result $\bar{V}$ as

$$
\bar{V}=\overline{V_{0}}-\overline{\bar{Z}}\left[\overline{\bar{H}}\left(\overline{\bar{M}}^{T} \overline{V_{0}}\right)\right] .
$$

Since the LU decomposition of $\overline{\bar{Y}}$ is already available, solution of (22) comes at a much smaller cost than solving the system starting from scratch. Also, the matrix $\overline{\bar{H}}$ in (23) has a small size. It is actually a scalar, if only one decap is to be modified. The presented approach can be applied when modifying a few decaps or the geometry of the power/ground planes. It is, therefore, more general than the approaches in [7] and [16], which only apply when a decap is completely removed or added to the PDN. The Woodbury formula has also been previously applied to efficiently create augmented equivalent circuit models of microwave devices [17].

Note the difference between this method and the calculation of the sensitivity of the solution discussed in Section II. Using the Woodbury formula, we can exactly (within numerical limits) calculate the impedance profile of networks with changes on a few parameters very efficiently. The sensitivity, on the other hand, just provides a feeling of how the impedance would change if there were a small change in the parameters. The Woodbury formula works in case of both small and large changes. One immediate application of this approach would be to find out how the impedance profile changes with the addition of decaps. A systematic optimization algorithm, as in Section III, also requires simulation of multiple similar designs at each iteration; hence, it can benefit from 
using the Woodbury formula assuming only a few parameters are modified [i.e., when the cost of (22)-(24) is smaller than directly solving (20)].

\section{A. Numerical Example for Fast Design Space Exploration}

The large-change sensitivity approach is applied on the example considered in Fig. 3, where a decap with $C=100 \mathrm{nF}$, equivalent series inductance $=0.2 \mathrm{nH}$, and equivalent series resistance $=10 \mathrm{~m} \Omega$ is placed in one corner of the board. The presence of the decap changes the impedance profile significantly as shown in Fig. 6. The dashed line represents the simulation result without the decap. The solid line is the simulation with the addition of the decap. The "+" markers correspond to the simulation result built upon the "without decap" case, where the effect of the decap is exactly captured using the Woodbury formula. For this example, since only one decap is being added, the matrices $\overline{\bar{U}}$ and $\overline{\bar{M}}$ reduce to vectors. The $\overline{\bar{U}}$ vector is chosen to have one nonzero element equal to 1 at the node corresponding to the location of the capacitor. The $\overline{\bar{M}}$ vector then has one nonzero element equal to the admittance of the capacitor at the same location.

In this example, a problem with 120000 nodes provided a speedup of 17 times. As the number of unknowns in typical PDN simulations is in the order of millions, there will be a greater advantage in using the large-change sensitivity approach.

\section{B. Measurement Verification for Fast Design Space Exploration}

Decoupling capacitors do not effectively provide isolation at gigahertz frequencies because of the parasitic inductance. One approach to provide reduction in noise coupling between various areas of a power plane is using power islands [18], as shown in Fig. 7. This test structure is very similar to the one presented in [18] except its dimensions. The dielectric thickness of the test structure is $117 \mu \mathrm{m}$, the dielectric constant is 4.1 , and the loss tangent is 0.02 . In order to find the optimum location of the connecting bridge, various simulations need to be performed. For the given example, four different scenarios have been simulated, where a single $0.5-\mathrm{mm}$ bridge has been connected to the center of the right, top, left, or bottom edge of the power island. Fig. 7 shows the case, where the bridge is connected to the right edge. All of these cases have been simulated using the large-change sensitivity algorithm; hence, required only one LU decomposition. The simulation results agree well with measurements as shown in Fig. 8. The simulation results can be used to decide where to place the conducting bridge for given specifications such as the required target transfer impedance over a certain frequency range. In this example, the conducting bridge has been modeled as the inductance of a short section of a microstrip line. The simulation is first performed with no bridge at all to find the LU decomposition of the nodal admittance matrix. Next, the conducting bridge is included in the simulation by connecting an impedance $Z$, which corresponds to the inductance of the bridge, across the relevant nodes depending on which case is being simulated. The addition of a series impedance $Z$ results in the addition of four different elements to the nodal admittance matrix as in (9). This addition is implemented by choosing the $\overline{\bar{U}}$ vector to have one nonzero element equal to 1 and another nonzero element equal to -1 at the two nodes, where the impedance is inserted. The $\overline{\bar{M}}$ vector then has the elements $1 / Z$ and $-1 / Z$ at these two nodes.

\section{CONCLUSION}

This paper presented new algorithms for sensitivity calculations and optimization of system-level PDNs. The sensitivity of the input impedance of a power/ground plane pair is calculated at negligible cost using the adjoint system method. The sensitivity information is useful in many ways: 1) it is required by gradient-based optimization algorithms; 2) it provides a frequency range, where the input impedance of the PDN is insensitive to a certain capacitance; and 3 ) it indicates which decaps could be causing most problems in terms of variability of the PDN impedance. A minimax optimization algorithm was then built on this sensitivity algorithm. The optimization algorithm minimizes the maximum deviation from the target impedance. Using a frequency-dependent target impedance profile, the multiobjective optimization in the minimax algorithm can be quite useful, when the impedance at a particular frequency (e.g., corresponding to one of the clock harmonics) needs to be decreased.

A large sensitivity approach has also been presented for the PDNs based on the Woodbury formula. It allows the simulation of similar designs with changes in only a few parameters, an order of magnitude faster. Hence, the presented large-change sensitivity approach is very useful in simulation of what-if scenarios, which are typical in power integrity analysis.

\section{REFERENCES}

[1] M. Leone, "The radiation of a rectangular power-bus structure at multiple cavity-mode resonances," IEEE Trans. Electromagn. Compat., vol. 45, no. 3, pp. 486-492, Aug. 2003.

[2] L. Smith, R. Anderson, D. Forehand, T. Pelc, and T. Roy, "Power distribution system design methodology and capacitor selection for modern CMOS technology," IEEE Trans. Adv. Packag., vol. 22, no. 3, pp. 284 291, Aug. 1999.

[3] X. Hu, W. Zhao, P. Du, Y. Zhang, A. Shayan, C. Pan, A. E. Egin, and C.-K. Cheng, "On the bound of time-domain power supply noise based on frequency-domain target impedance," in Proc. 11th Int. Workshop Syst. Level Interconnect Prediction (SLIP 2009). New York, 2009, pp. 6976.

[4] Y. Shi, J. Xiong, C. Liu, and L. He, "Efficient decoupling capacitance budgeting considering operation and process variations," IEEE Trans. Comput.-Aided Design Integr. Circuits Syst., vol. 27, no. 7, pp. 12531263, Jul. 2008.

[5] T. Hubing, J. Drewniak, T. Van Doren, and D. Hockanson, "Power bus decoupling on multilayer printed circuit boards," IEEE Trans. Electromagn. Compat., vol. 37, no. 2, pp. 155-166, May 1995.

[6] K. Bharath, E. Engin, and M. Swaminathan, "Automatic package and board decoupling capacitor placement using genetic algorithms and $\mathrm{m}$ fdm," in Proc. 45th ACM/IEEE Des. Autom. Conf. (DAC 2008), Jun., pp. 560-565.

[7] J. Chen and L. He, "Efficient in-package decoupling capacitor optimization for i/o power integrity," IEEE Trans. Comput.-Aided Design Integr. Circuits Syst., vol. 26, no. 4, pp. 734-738, Apr. 2007.

[8] H. Zheng, B. Krauter, and L. Pileggi, "On-package decoupling optimization with package macromodels," in Proc. IEEE Custom Integr. Circuits Conf. 2003, Sep., pp. 723-726. 
[9] H. Su, S. Sapatnekar, and S. Nassif, "Optimal decoupling capacitor sizing and placement for standard-cell layout designs," IEEE Trans. Comput.Aided Design Integr. Circuits Syst., vol. 22, no. 4, pp. 428-436, Apr. 2003.

[10] O. Ramahi, V. Subramanian, and B. Archambeault, "A simple finitedifference frequency-domain (FDFD) algorithm for analysis of switching noise in printed circuit boards and packages," IEEE Trans. Adv. Packag., vol. 26, no. 2, pp. 191-198, May 2003.

[11] T. Tarvainen, "Simplified modeling of parallel plate resonances on multilayer printed circuit boards," IEEE Trans. Electromagn. Compat., vol. 42, no. 3, pp. 284-289, Aug. 2000.

[12] M. Swaminathan and A. E. Engin, Power Integrity Modeling and Design for Semiconductors and Systems. Upper Saddle River, NJ: PrenticeHall, 2007.

[13] J. Vlach and K. Singhal, Computer Methods for Circuit Analysis and Design. New York: Wiley, 1993.

[14] R. Brayton, S. Director, G. Hachtel, and L. Vidigal, "A new algorithm for statistical circuit design based on quasi-newton methods and function splitting," IEEE Trans. Circuits Syst., vol. CAS-26, no. 9, pp. 784-794, Sep. 1979.

[15] W. H. Press, S. A. Teukolsky, W. T. Vetterling, and B. P. Flannery, Numerical Recipes in C: The Art of Scientific Computing. New York: Cambridge Univ. Press, 1992.

[16] J. Zhao and O. Mandhana, "A fast evaluation of power delivery system input impedance of printed circuit boards with decoupling capacitors," in Proc. IEEE 13th Top. Meet. Electr. Perform. Electron. Packag., 2004, Oct., pp. 111-114.

[17] D. Paul, M. Nakhla, R. Achar, and A. Weisshaar, "Broadband modeling of high-frequency microwave devices," IEEE Trans. Microw. Theory Tech., vol. 57, no. 2, pp. 361-373, Feb. 2009.

[18] W. Cui, J. Fan, H. Shi, and J. Drewniak, "Dc power bus noise isolation with power islands," in Proc. IEEE Int. Symp. Electromagn. Compat. (EMC 2001), vol. 2, pp. 899-903.

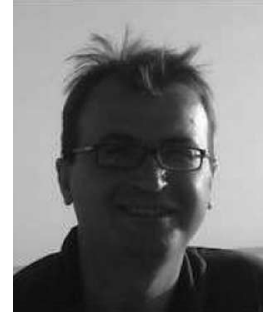

A. Ege Engin (M'05) received the B.S. and M.S. degrees in electrical engineering from Middle East Technical University, Ankara, Turkey and the University of Paderborn, Paderborn, Germany, in 1998 and 2001, respectively, and the Ph.D. degree (summa cum laude) from the University of Hannover, Hannover, Germany, in 2004.

He was a Research Engineer at the Fraunhofe Institute for Reliability and Microintegration, Berlin, Germany. From 2006 to 2008, he was an Assistant Research Director of the Microsystems Packaging Research Center at the Georgia Institute of Technology. He is currently an Assistant Professor in the Electrical and Computer Engineering Department, San Diego State University, San Diego, CA. He has authored or coauthored more than 70 papers published in various international journals and conference proceedings in the areas of signal and power integrity modeling and simulation, a book Power Integrity Modeling and Design for Semiconductors and Systems (Prentice-Hall, 2007) and holds one patent and three patent applications.

Dr. Engin is the recipient of the Semiconductor Research Corporation Inventor Recognition Award in 2009. He is also a recipient of the Outstanding Poster Paper Award in the Electronic Components and Technology Conference 2006 for his publications, the Best Paper Award Finalist in the Board-Level Design Category at DesignCon 2007, and the Best Paper of the Session Award in the International Microelectronics and Packaging Society Advanced Technology Workshop on RF and Microwave Packaging 2009. 